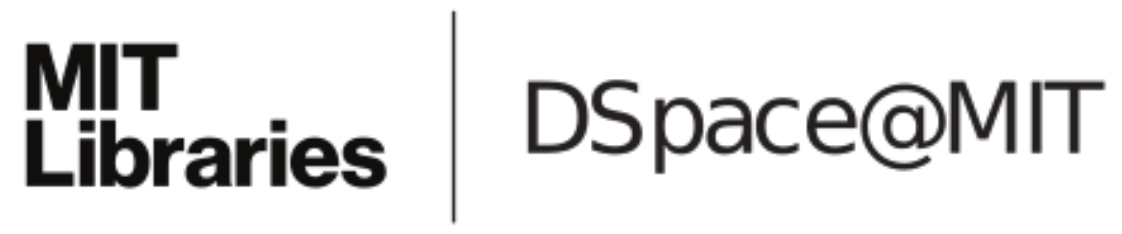

\author{
MIT Open Access Articles
}

Nano-beam and nano-target effects in ion radiation

The MIT Faculty has made this article openly available. Please share how this access benefits you. Your story matters.

Citation: Yang, Yang, Yong Gang Li, Michael P. Short, Chung-Soo Kim, Karl K. Berggren, and Ju Li. “Nano-Beam and Nano-Target Effects in Ion Radiation.” Nanoscale 10, no. 4 (2018): 15981606.

As Published: http://dx.doi.org/10.1039/c7nr08116b

Publisher: Royal Society of Chemistry

Persistent URL: http://hdl.handle.net/1721.1/120357

Version: Original manuscript: author's manuscript prior to formal peer review

Terms of use: Creative Commons Attribution-Noncommercial-Share Alike 


\title{
Nano-beam and nano-target effects in ion
} radiation $\dagger$

Cite this: DOI: $10.1039 / \mathrm{c} 7 \mathrm{nr} 08116 \mathrm{~b}$

Received 31st October 2017

Accepted 11th December 2017

DOI: $10.1039 / \mathrm{c} 7 \mathrm{nr} 08116 \mathrm{~b}$

\author{
Yang Yang, ${ }^{a}$ łonggang Li, ${ }^{\text {a,b,c }}$ Michael P. Short, ${ }^{a}$ Chung-Soo Kim, ${ }^{d}$ Karl K. Berggren ${ }^{d}$ \\ and Ju Li (iD *a,e
}

rsc.li/nanoscale

Full three dimensional (3D) simulations of ion implantation are necessary in a wide range of nanoscience and nanotechnology applications to capture the increasing effect of ion leakage out of surfaces. Using a recently developed 3D Monte Carlo simulation code IM3D, we first quantify the relative error of the 1D approach in three applications of nano-scale ion implantation: (1) using a nano-beam for nitrogen-vacancy (NV) center creation, (2) implantation of nanowires to fabricate $p-n$ junctions, and (3) irradiation of nano-pillars for small-scale mechanical testing of irradiated materials. Because the 1D approach fails to consider the exchange and leakage of ions from boundaries, its relative error increases dramatically as the beam/target size shrinks. Lastly, the "Bragg peak" phenomena, where the maximum radiation effect occurs at a finite depth away from the surface, rely $y_{2}$ on the assumption of broad beams. We discovered a topological transition of the pointdefect or defect-cluster distribution isosurface when one varies the beam width, in agreement with the previous focused helium ion beam irradiation experiment. We conclude that full 3D simulations are necessary if either the beam or the target size is comparable or below the SRIM longitudinal ion range.

Nanoscale ion implantation represents an expanding, interdisciplinary field that combines radiation effects with nanoengineering to control matter at the atomic level. In doing $\mathrm{se}_{2}$ it offers the potential to create novel nano-devices such as quantum computers, ${ }^{1,2}$ magnetometers, ${ }^{3}$ nanowire $\mathrm{pm}_{\mathbf{2}}$ junctions ${ }^{4}$ etc. In particular, ion implantation enables more precise control of the spatial distribution and concentration of

\footnotetext{
${ }^{a}$ Department of Nuclear Science and Engineering, Massachusetts Institute of Technology, Cambridge, Massachusetts 02139, USA. E-mail: liju@mit.edu

${ }^{b}$ Key Laboratory of Materials Physics, Institute of Solid State Physics, Chinese Academy of Sciences, Hefei, 230031, China

${ }^{c}$ University of Science and Technology of China, Hefei, 230026, P. R. China

${ }^{d}$ Department of Electrical Engineering and Computer Science, Massachusetts Institute of Technology, Cambridge, Massachusetts 02139, USA

${ }^{e}$ Department of Materials Science and Engineering, Massachusetts Institute of Technology, Cambridge, Massachusetts 02139, USA

$\dagger$ Electronic supplementary information (ESI) available. See DOI: 10.1039/
} c7nr08116b dopants/vacancies, making it highly desirable for the fabrication of nano-devices reproducibly. However, confidently taking advantage of it hinges upon the accurate and precise knowledge of the spatial distribution of point defects (including dopants, vacancies, and self-interstitials) created by ion implantation, which is often predicted by Monte Carlo simulations. This work aims to explore two primary but uncategorized nano-sized effects that may limit the accuracy of the current widely-used simulation methods-significantly.

Stopping and range of ions in matter (SRIM), ${ }^{5}$ a popular tool for calculating ion range, straggling, sputtering, and primary radiation damage, is the workhorse of the ion beam community. ${ }^{1,2,6,7}$ Although SRIM actually performs certain 3D simulations and record full 3D coordinates for all collision events (available for collection from the files), its 1D output, which simulates the effects of a broad beam on single- or multi-layered materials, is most widely used because of its simplicity. Suppose there is a distribution of some point defect in $3 \mathrm{D}$, denoted as $F(x, y, z)$, then the 1D output addresses the integral point defect distribution along the $z$-axis (depth):

$$
f(z) \equiv \iint_{-\infty}^{\infty} F(x, y, z) \mathrm{d} x \mathrm{~d} y
$$

This approach is sketched in Fig. 1a. $f(z)$ becomes the complete mathematical descriptor in the following two limiting cases: (1) when an ion beam of beam-width $\sigma$ is rastered uniformly across a sample of a size $L$, where $L$ is much larger than the lateral straggling range $L_{\mathrm{S}}$ of the ions ( $L_{\mathrm{S}}$ usually ranges from a few nanometers to a few microns). (2) When the beam is not rastered, and a broad beam $\sigma \gg L_{\mathrm{S}}$ and $L \gg L_{\mathrm{S}}$ (see Fig. 1bI) is used instead. Traditional ion irradiation employs a beam width $\sigma$ on the order of millimeters.

Ion irradiation techniques and approaches, however, have vastly improved with the proliferation of nanotechnology. First, ion beam spot sizes have reached sub-micron sizes ${ }^{8}$ for $\mathrm{MeV}$ proton beams and sub-nanometer sizes ${ }^{9}$ for $\mathrm{keV}$ focused ion beams (He, $\mathrm{Ne}$ etc.), either by new implantation systems $^{8-12}$ or nano-scale apertures. ${ }^{1,13-16} \mathrm{MeV}$ proton nanobeams (beam widths as low as $14 \mathrm{~nm}$ (ref. 10) have been used 


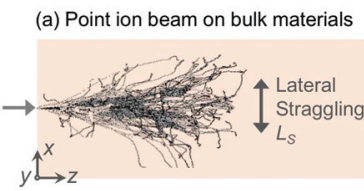

(b)

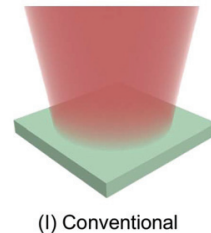

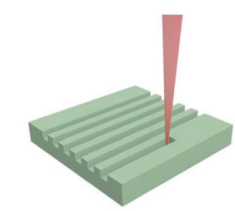

(II) Nano beam
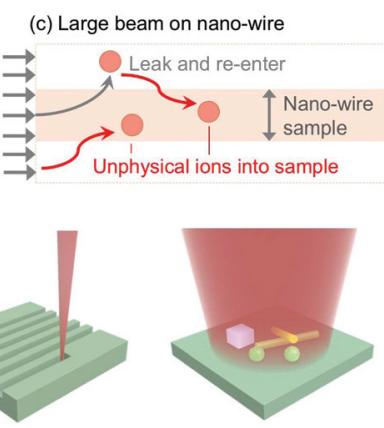

(III) Nano target
Fig. 1 (a) Illustration of the 1D simulation approach and ion trajectories as created by SRIM. ${ }^{5}$ The lateral straggling is integrated by the 1D approach, smearing out 3D details in the final results. (b) Schematic illustration of: (I) conventional ion implantation ( $\sigma \gg L_{S}$ and $L \gg L_{S}$ ); (II) nano-beam ( $\sigma \sim L_{\mathrm{S}}$ or $\left.\sigma<L_{\mathrm{S}}\right)$; and (III) nano-target ( $L \sim L_{\mathrm{S}}$ or $\left.L<L_{\mathrm{S}}\right)$ scenarios. SRIM's 1D approach can only simulate (I) correctly. With an input file listing all ions' beginning positions, it is possible but complicated to simulate (II) using SRIM, while it cannot simulate (III). (c) An example of the nano-target effect: large beam ion irradiation of a nanowire. The 1D approach creates unphysical ions which enter the nano-sized sample, with their trajectories highlighted in red.

for writing nano-scale patterns with high aspect ratios. ${ }^{8,10}$ Nano-sized ion beams in radiobiological research also facilitate accurate studies of the effects of irradiation at the cellular and subcellular levels. ${ }^{17}$ Nano-scale apertures, such as modified atomic force microscope tips, ${ }^{15}$ mica masks with nanochannels, ${ }^{14}$ silicon masks with atomic layer deposition (ALD) filled slits, ${ }^{1}$ electron beam lithography resist masks ${ }^{13}$ etc., have been used to focus the ion beam as much as possible in order to realize the nanometer level control of the location of the ion beam. For these situations, the 1D simulation approach leads to incorrect predictions and misunderstandings.

Various advanced codes have been developed to enable full3D simulations, including iradina, ${ }^{18}$ TRI3DYN, ${ }^{19} \mathrm{IM}^{12} \mathrm{D}^{20}$ and Corteo $2 \mathrm{D} / 3 \mathrm{D}$ module. ${ }^{21}$ IM3D is equipped with the finite element triangular mesh (FETM) method and parallel computation, thus it can simulate ion radiation in arbitrary target shapes at high efficiency. In this letter, we utilize IM3D to quantify the effect of nanoscale ion beam sizes and target features on the resulting primary radiation damage and other point defect distributions. The size effect can be further divided into the nano-beam and nano-target effects, as illustrated in Fig. 1b. These effects may also manifest together, as the nano-beam may strongly interact with the target to evolve nanoscale features. Finally, we will also address the necessity of full-3D simulations when considering multi-defect reactions, and unravel the 3D topological evolution of the point defect distribution isosurface as the beam size varies.

Consider an ion beam of flux $\phi$ (ions per area per second) and width $\sigma$. Since the $1 \mathrm{D}$ representation of such a beam is smeared over $x$ and $y$, it is equivalent to simulating a beam with a much larger width $\sigma^{\prime} \gg \sigma$, creating unphysical ion sources outside the real nano-sized beam. A limiting case is a point beam, $\sigma \rightarrow 0$ : most of the ions will scatter away from the original entrance line, while the 1D output takes all ions 'back' by smearing. For nano-targets, as shown in Fig. $1 \mathrm{~b}$ and c, error also results from physically impossible re-entrant ions. Here we present four simulations to illustrate the importance of the nano-beam and nano-target effects. Simulation parameters for the three cases, (1) Nitrogen Vaeaney (NV) center creation in diamond, (2) nanowire doping, and (3) irradiation of nanopillars for mechanical testing, as well as (4) a confirmatory focused helium ion beam (FHIB) experiment, are summarized in Table 1.

Implanting ${ }^{15} \mathrm{~N}^{+}$ions into diamond creates NV centers. Our first example is the case of a thick mask with nano-pinholes to produce NV center networks for quantum computation, ${ }^{1,13-15}$ an idealized illustration of the nano-beam effect. This masking technique, shown schematically in Fig. 2a, enables highly accurate control of the implantation location(s). Ion ranges, implanted ion concentrations, and vacancy concentrations are important factors for understanding the depth and yield of NV center creation. However, the 1D approach will significantly overestimate the effective dose and the yield of NVs for pinhole masks.

The NV center creation case illustrated in Fig. 2a was modeled using both the SRIM 1D approach and IM3D to highlight the differences in the results. The mask was made thick enough to be impermeable to the implanting ${ }^{15} \mathrm{~N}^{+}$ions, with a square shaped pinhole. The total injected ion and total vacancy concentrations were then calculated at the centerline of the channel, to compare the 1D and full-3D approaches. Numerically, these values were calculated by Monte Carlo

Parameters

\begin{tabular}{|c|c|c|c|c|c|c|c|c|c|}
\hline Case & Ion type & Ion energy & Target & $\begin{array}{l}\text { Density } \\
\left(\mathrm{g} \mathrm{cm}^{-3}\right)\end{array}$ & $\begin{array}{l}\text { Displacement } \\
\text { threshold } \\
\text { energy }^{5}(\mathrm{eV})\end{array}$ & $\begin{array}{l}\text { Lattice } \\
\text { energy } \\
(\mathrm{eV})\end{array}$ & $\begin{array}{l}\text { Surface } \\
\text { energy } 5 \\
(\mathrm{eV})\end{array}$ & $\begin{array}{l}\text { SRIM longitudinal } \\
\text { ion range } \\
\text { (along the } z \text { axis) }\end{array}$ & $\begin{array}{l}\text { Number of } \\
\text { ions per } \\
\text { simulation }\end{array}$ \\
\hline NV center creation & ${ }^{15} \mathrm{~N}^{+}$ & $100 \mathrm{keV}$ & $\mathrm{C}$ & 3.53 & 28 & 3 & 7.41 & $116.2 \mathrm{~nm}$ & $10^{9}$ \\
\hline Irradiated nano-pillars & ${ }^{1} \mathrm{H}^{+}$ & $1.1 \mathrm{MeV}$ & $\mathrm{Cu}$ & 8.92 & 25 & 3 & 3.52 & $7.8 \mu \mathrm{m}$ & $2 \times 10^{7}$ \\
\hline FHIB experiment & ${ }^{4} \mathrm{He}^{+}$ & $35 \mathrm{keV}$ & $\mathrm{Si}$ & 2.33 & 15 & 2 & 4.7 & $318.3 \mathrm{~nm}$ & $10^{6}$ \\
\hline
\end{tabular}


(a)

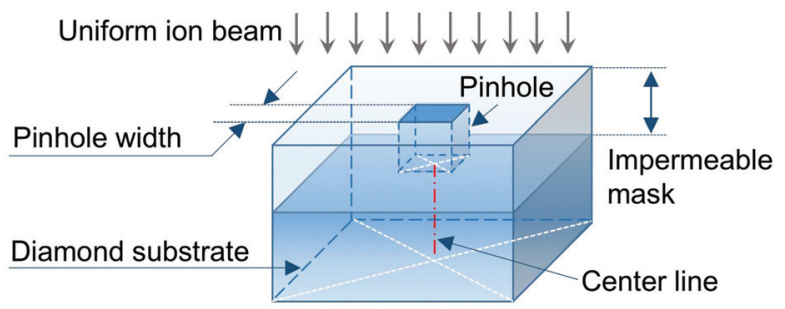

10

(b)

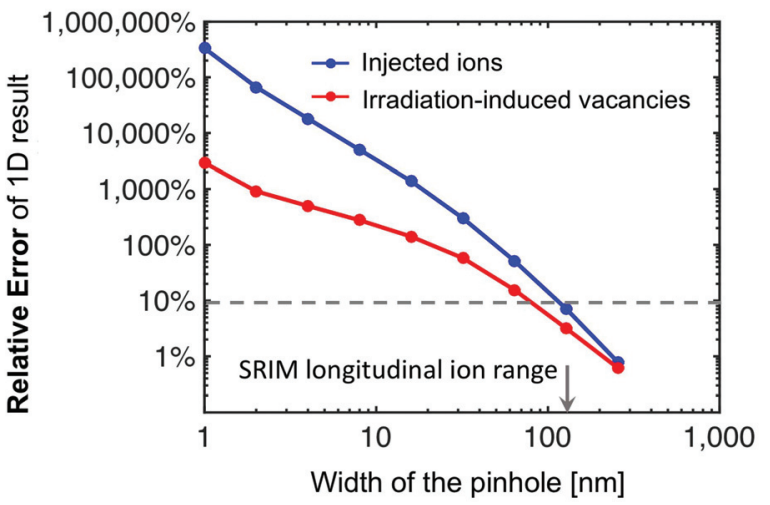

Fig. 2 Schematic and quantification of the nano-beam effect in the case of NV center creation. (a) Diagram showing the case of targeted NV center creation. (b) Relative error of the 1D approach compared to the full 3D approach. Both statistics are integrated in a $1 \mathrm{~nm}$-radius cylindrical volume along the centerline, shown in red in (a). sampling in a test-cylindrical volume with a radius of $1 \mathrm{~nm}$ at the centerline, shown as a red line in Fig. 2a. The relative error of the $1 \mathrm{D}$ approach is defined as:

$$
\text { Relative error } \equiv\left|\frac{T_{1 \mathrm{D}}-T_{\mathrm{IM} 3 \mathrm{D}}}{T_{\mathrm{IM} 3 \mathrm{D}}}\right| \times 100 \%
$$

where $T_{1 \mathrm{D}}$ and $T_{\mathrm{IM} 3 \mathrm{D}}$ represent the total number of a kind of point defect counted in the volume of interest (i.e. the test cylinder in this case) by the $1 \mathrm{D}$ approach and IM3D, respectively. The variation of the relative error of the $1 \mathrm{D}$ approach versus the width of the pinhole in the mask is plotted in Fig. 2b. Note that the relative error is still $10 \%$ even when the hole width is $128 \mathrm{~nm}$, which is even larger than the SRIM longitudinal ion range $(116 \mathrm{~nm})$, i.e., the average penetration depth by the $1 \mathrm{D}$ approach. In other words, with any beam width smaller than the SRIM longitudinal ion range, very significant errors $(>10 \%)$ are created using the 1D simulation approach. When the pinhole is $1 \mathrm{~nm}$ wide, the relative overestimation of the injected ions via the 1D approach can be as high as $200000 \%$, since most ions scatter away from the cylinder in the center and exit the NV center volume. The error of the vacancy concentration is 1-2 orders of magnitude smaller than that of the injected ions.

Alternately, the nano-beam effect on a bulk isotropic sample can be predicted by a convolution of $F(x, y, z)$ and beam shape function (see theories of Furukawa ${ }^{22}$ and Runge ${ }^{23}$ ), which will be discussed in detail later. This convolution method has been extensively applied in the studies of e-beam lithography ${ }^{24} /$ e-beam-induced deposition ${ }^{25}$ especially the proximity effect, ${ }^{26-28}$ which describes the undesired exposure by scattering of electrons. The proximity effect of the e-beam is similar to the nano-beam effect of ion-implantation.

Introduction of dopants, vacancies, or dislocations into nanomaterials by ion irradiation has been an extremely useful method for designing novel devices, ${ }^{29-36}$ such as the use of $\mathrm{Ar}^{+}$ irradiation to tune the electrical conductivity of single-walled carbon nanotubes (SWCNTs). ${ }^{37}$ Nano-targets usually have sizes close to or less than the penetration range of the ions. For such a nano-sized target, utilizing the $1 \mathrm{D}$ approach will not only create unphysical ion sources outside the real beam, but will also fail to consider the effect of irreversible ion leakage outside the material boundary, i.e., once an ion leaves the sample, it generally cannot come back into the sample. In the 1D approach, however, it may still re-enter as illustrated in Fig. 1c.

Previously, ion implantation on nano-targets (nanowires $^{38-41}$ and FinFETs ${ }^{42}$ ) has been modelled by several researchers. In our second simulation, we address a special case of ion radiation on nanowires, i.e., head-on ion implantation experiment for making axial $\mathrm{p}-\mathrm{n}$ junctions in silicon nanowires (NWs), ${ }^{4}$ to illustrate the target size effects on point defect distributions. In the experiment, silicon nanowires were first grown perpendicular to a substrate, and post-growth ion implantation $\left({ }^{11} \mathrm{~B}^{+}\right.$as the electron acceptor, and ${ }^{31} \mathrm{P}^{+}$as the electron donor) was applied with the ion-incident direction perpendicular to the substrate, as shown in Fig. 3a. To replicate this experiment, $180 \mathrm{keV}{ }^{31} \mathrm{P}^{+}$implantation in a $500 \mathrm{~nm}$ long single silicon nanowire was simulated using a randomly distributed large beam source $(\sigma \gg L)$. Averaging over the nanowire cross-section, the injected-ion and vacancy distributions are plotted along the axes of the nanowires for different radii $R_{\mathrm{NW}}$, as shown in Fig. $3 \mathrm{~b}$ and c. As $R_{\mathrm{NW}}$ becomes smaller, the amplitude and peak depth of both the ion and vacancy curves decrease dramatically due to ion losses at nanowire boundaries. The vacancy peak shifts to a shallower depth (close to the entrance of the beam) more quickly than the ion peak, as shown in Fig. 3b and c. The relative error of the SRIM $1 \mathrm{D}$ output compared to the full-3D approach is plotted in Fig. 3d as solid lines. The volume of interest for calculation of relative errors in this case is the whole nanowire. The radiation damage, measured in vacancies per ion, is much less sensitive to the nanowire radius than the implanted ion distribution. The relative error of the implanted ions is above $20 \%$ even when the nanowire is $500 \mathrm{~nm}$ in radius (i.e. the diameter is 4.2 times the SRIM longitudinal ion range). Thus, the full-3D approach is absolutely necessary to accurately model radiation damage and injected ion distributions in nanowires.

In the third example, we address a popular method of nano-mechanical testing of ion irradiated materials to study radiation effects on their mechanical behavior. ${ }^{43,44}$ As an extension of the nano-target effect, we use a recent experiment ${ }^{44}$ as an example for the edge effect when irradiating a large sample $\left(L \gg L_{\mathrm{S}}\right)$ with a uniformly rastered beam. 
(a)

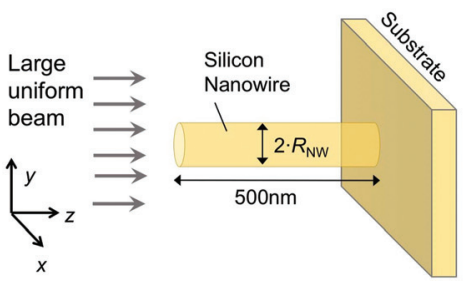

(c)

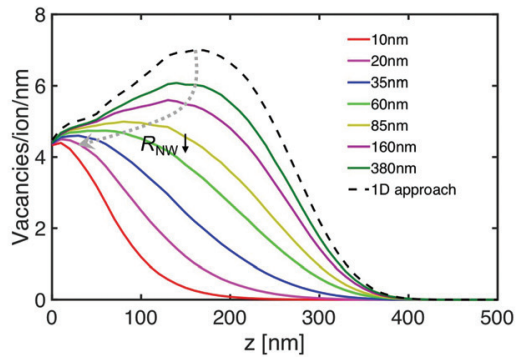
twice the SRIM ange.
As shown in Fig. $4 \mathrm{a}$, a $5 \mu \mathrm{m}$ thick lamella was cut using a focused ion beam (FIB) before irradiation. After acceleratorbased irradiation with $1.1 \mathrm{MeV} \mathrm{H}^{+}$ions, one side of the lamella was machined by FIB again to create a pillar (enclosed by bluish dashed-line in Fig. 4a), with the diameter ranging from $80 \mathrm{~nm}$ to $1500 \mathrm{~nm}$, for compression testing. Using the SRIM-based 1D approach, it was estimated that radiation damage of the pillars was $0.8 \pm 0.01$ displacements per atom (DPA) for the smaller samples, and $0.8 \pm 0.09$ DPA for the largest pillars. The errors are due to the dose variation along the $z$-axis. However, the 1D approach ignores the fact that ions leak from the edge (shown in Fig. 1c), along with unphysical ion sources created outside the edge. IM3D shows a large variation of radiation damage along the $x$-axis at the pillar top due to the edge effect, as shown in Fig. 4b. Although the dose asymptotically reaches 0.8 DPA further away from the edge, the relative error can be around $90 \%$ at the edge, and is still as high as $25 \%$ at $100 \mathrm{~nm}$ from the edge. Since the aspect ratio for the pillar varies from $3: 1$ to $5: 1$, the length of nano-pillars with $100 \mathrm{~nm}$ diameter for in situ TEM mechanical tests ranges from $300-500 \mathrm{~nm}$. Therefore, the whole length of small sized pillars suffers from significant edge dose attenuation effects. In order to have a pillar with a relatively uniform dose distribution, it is recommended that the top $400 \mathrm{~nm}$ of the pillar (i.e., $x \in[0,400]$, also shown in Fig. $4 \mathrm{c}$ ) should be removed before the compression test, while the fabrication processes of such pillars usually remove just less than $100 \mathrm{~nm}$ of the top to reduce the FIB-related taper.

Based on the three examples above, it is seen that full-3D modeling is critical for beam/target sizes below $k$ times the SRIM longitudinal ion range, where $k=5$ in our first two (b)

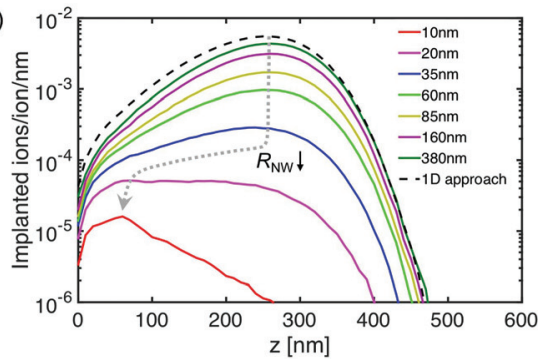

(d)

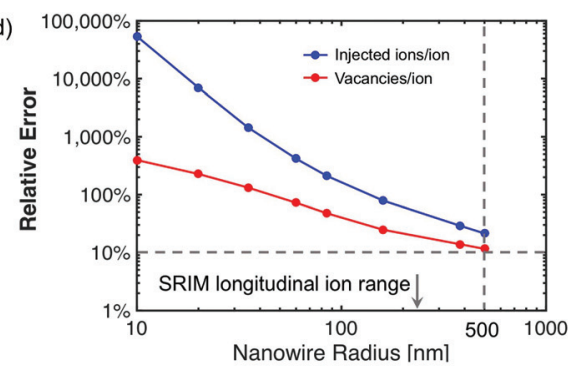

1

Fig. 3 (a) Schematic illustration of ion implantation of a nanowire. (b) Implanted ion distribution along the $z$-axis for different nanowire radii $R_{\mathrm{NW}}$. The amplitude of the curve decreases for smaller wires, while the peak position is almost fixed until $R_{\mathrm{NW}}<35 \mathrm{~nm}$. (c) Vacancy distribution along the wire for different $R_{\mathrm{NW}}$ values. Both the amplitude and peak depth of the curve decrease as the wire size shrinks. The grey-dotted-arrows in (b) and (c) represent the shifting trend of peaks' position and amplitude. (d) Relative error of the 1D approach, which is larger than $10 \%$ if $R_{\mathrm{NW}}$ is smaller than

examples. In fact, $k$ depends on ion mass, energy, target composition, structure etc. In addition, special attention should be paid to the edges even if irradiation is performed on a large sample, as shown in the third example. More details about eensiderations on this criterion are provided in ESI S2. $\dagger$

The spatial distribution of point defects calculated by Monte Carlo simulation is often used as an input for point kinetics $^{45}$ or cluster dynamics ${ }^{46}$ studies. Here we address another important problem with inappropriate use of the 1D simulation approach. Co-implantation, as well as dopant implantation together with radiation-induced vacancies, may lead to composite defects like divacancies, helium-vacancy clusters, which can play critical roles in material properties. Taking the divacancy (DV) as an example, suppose we want to know at which depth $\left(z_{\max }\right)$ the number of DVs is maximized. These presumably arise from the recombination of single vacancies, whose 3D and 1D distributions are denoted as $F_{\mathrm{vac}}(x, y, z)$ and $f_{\mathrm{vac}}(z)$, respectively. One approach is to multiply the $1 \mathrm{D}$ profiles, resembling binary recombination in chemical kinetics:

$$
\tilde{f}_{\mathrm{DV}}(z) \sim f_{\text {vac }}(z) f_{\text {vac }}(z)
$$

and then maximize

$$
\arg \max \tilde{f}_{\mathrm{DV}}(z)=\arg \max f_{\mathrm{vac}}(z)^{2}
$$

where arg max denotes the arguments of the maxima, i.e. the point at which the function value is maximized. The above is correct for a broad beam $(\sigma \sim \mathrm{mm})$. However, for the case of a narrow beam the equation above is conceptually wrong, because it ignores the lateral straggling effect (see 
1

(a)
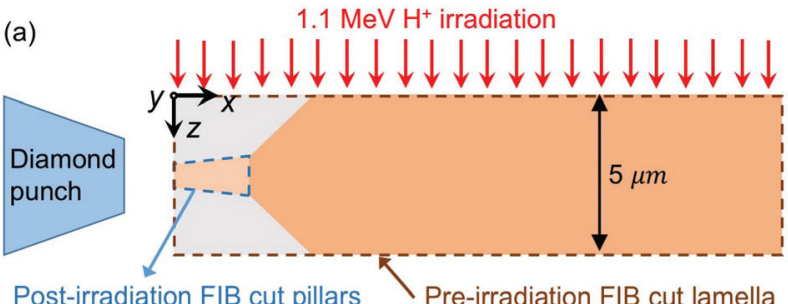

Post-irradiation FIB cut pillars for compression test

(b)

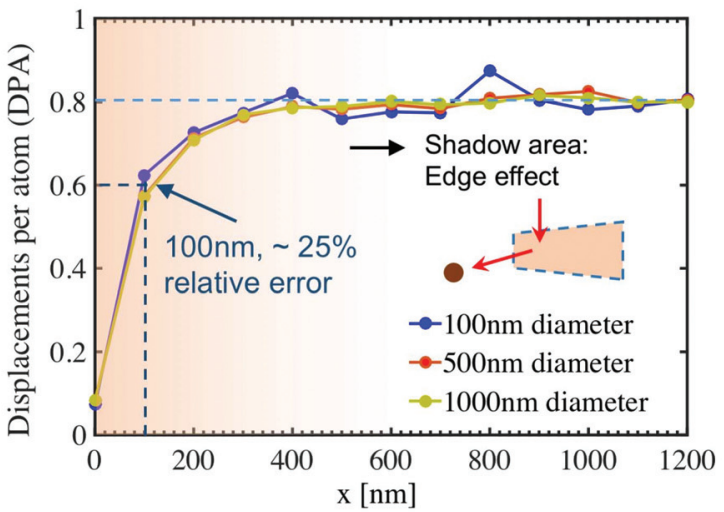

(c)

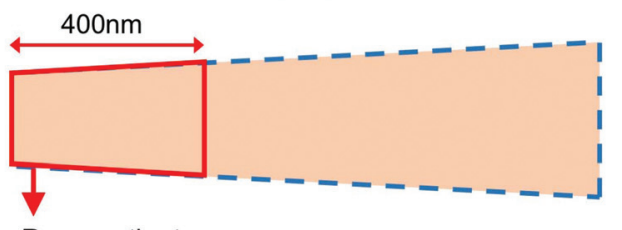

Remove the top

Fig. 4 (a) Illustration of the sample fabrication process and the irradiation configuration for irradiated nanopillar mechanical testing, modified from ref. 44. (b) Radiation dose in DPA along the $x$-axis in the pillar (100 nm, $500 \mathrm{~nm}$, and $1000 \mathrm{~nm}$ in diameter), showing strong edge effects in all cases. (c) Our 3D simulations suggest that removal of the top $400 \mathrm{~nm}$ of the pillar before compression testing should be performed, in order to obtain a uniform dose along the $x$-axis in the pillar. Note that only (b) and (c) share the same $x$ length scale.

Fig. 1a). This occurs when the $z$-dependent lateral straggling width causes $z_{\max }$ to be shifted to a shallower position than what eqn (4) predicts. The correct procedure is to compute the following 3D product:

$$
F_{\mathrm{DV}}(x, y, z) \sim F_{\mathrm{vac}}(x, y, z) F_{\mathrm{vac}}(x, y, z)
$$

and then project it into $1 \mathrm{D}$ using a double integral:

$$
f_{\mathrm{DV}}(z) \equiv \iint_{-\infty}^{\infty} F_{\mathrm{vac}}(x, y, z)^{2} \mathrm{~d} x \mathrm{~d} y
$$

$$
z_{\text {max }} \equiv \arg \max f_{\mathrm{DV}}(z)
$$

which generally gives a different result from (3). A more intuitive illustration is shown in Fig. 5, where $F_{\text {vac }}(x, y, z), f_{\text {vac }}(z)$, $\tilde{f}_{\mathrm{DV}}(z)$ and $f_{\mathrm{DV}}(z)$ generated by a $100 \mathrm{keV}{ }^{15} \mathrm{~N}^{+}$ion Gaussian nanobeam $(\sigma=5 \mathrm{~nm})$ in diamond are plotted and compared. Using the $1 \mathrm{D}$ approach, $\tilde{f}_{\mathrm{DV}}(z)$ renders a wrong result of $z_{\max }=$ $103.5 \mathrm{~nm}$, while $f_{\mathrm{DV}}(z)$ predicts two peaks: $z_{\max 1}=19.5 \mathrm{~nm}$ and $z_{\max 2}=37.5 \mathrm{~nm}$.

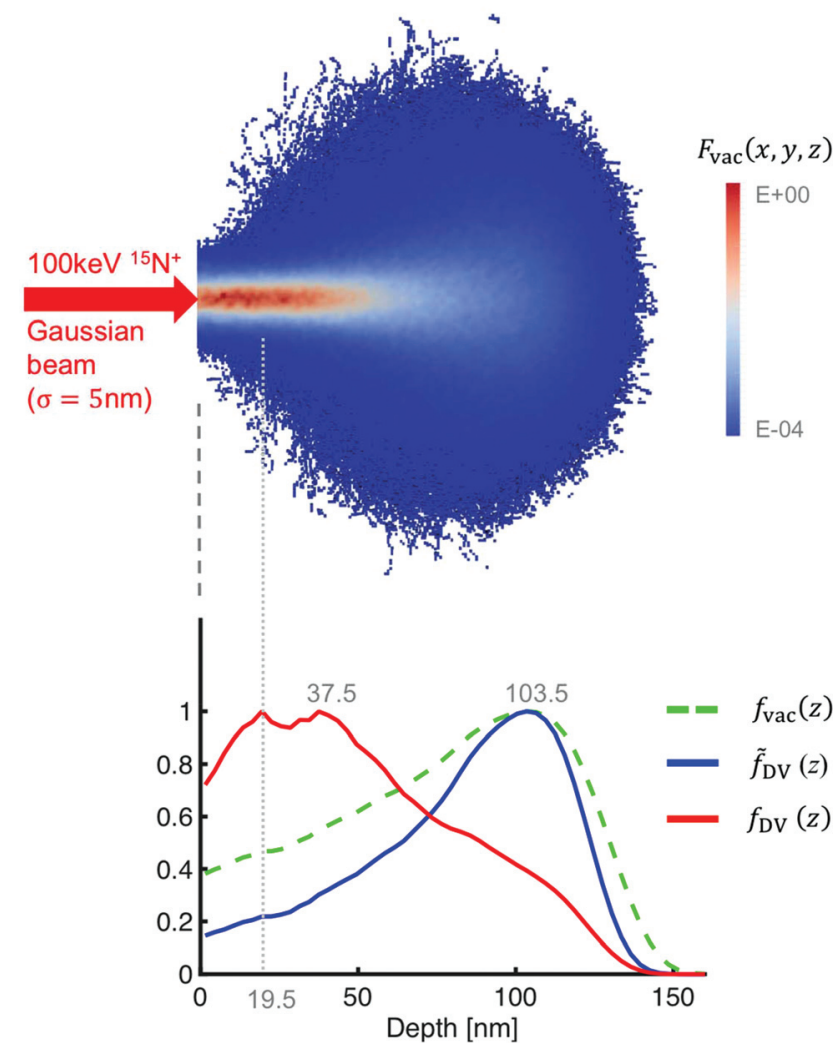

1

Fig. 5 An example showing that using the 1D approach will predict incorrect distributions of composite defects. (1) Single vacancy distribution in 3D, $F_{\mathrm{vac}}(x, y, z)$; (2) single vacancy distribution in 1D, $f_{\mathrm{vac}}(z)$, calculated using eqn (1); (3) unphysical divacancy (DV) distribution predicted by the $1 \mathrm{D}$ approach, $\tilde{f}_{\mathrm{DV}}(z)$, renders a single peak at $103.5 \mathrm{~nm}$; (4) DV distribution predicted by the full 3D approach, $f_{\mathrm{DV}}(z)$, gives two peaks (19.5 $\mathrm{nm}$ and $37.5 \mathrm{~nm}$, respectively), much shallower than the result of the 1D approach. The first peak of $f_{\mathrm{DV}}(z)$ results from the peak in $F_{\mathrm{vac}}(x, y$, $z$ ) (shown by a light-grey vertical dotted line), while the second peak is more of an integral effect. All defect distributions have been normalized (concentration at peak $=1$ ).

To see how the 3D distributions of point defects evolve as $\sigma$ varies, we visualize the injected ${ }^{15} \mathrm{~N}^{+}$density $F_{\text {ion }}(x, y, z)$, vacancy density $F_{\text {vac }}(x, y, z)$, and their product $F_{\text {ion }}(x, y, z) F_{\text {vac }}(x, y$, $z$ ) in $3 \mathrm{D}$ (Fig. 6b). This last term is proportional to the injected ion and vacancy reaction rate, an important quantity for the study of defect evolution and swelling. ${ }^{45}$ Isosurfaces of the half maximum (IHM), a 3D analog to the full-width-at-halfmaximum (FWHM), are computed for $F_{\text {ion }}(x, y, z)$ and $F_{\text {vac }}(x, y, z)$.

Even for a point beam source $(\sigma \rightarrow 0), F_{\text {ion }}(x, y, z)$ and $F_{\text {vac }}(x$, $y, z)$ occupy $3 \mathrm{D}$ domains. Such scattering-induced beam spreading/blurring is similar to the blurring of an image by an optical aperture, except that there is no phase interference. By making this optical analogy, the isosurface of the half maximum (IHM) is just like an Airy disk, but in the 3D space. The IHM of $F_{\text {ion }}(x, y, z)$ is a small sphere at the end of the tail. It is surprising that $\operatorname{IHM}\left(F_{\text {vac }}\right)$ is a short, sharp 'needle,' and does not overlap with $\operatorname{IHM}\left(F_{\text {ion }}\right)$. This is significantly different from the well-known 1D profiles, where the Bragg peak of the vacancy distribution (a 1D concept, pointed out in Fig. 6e) is 


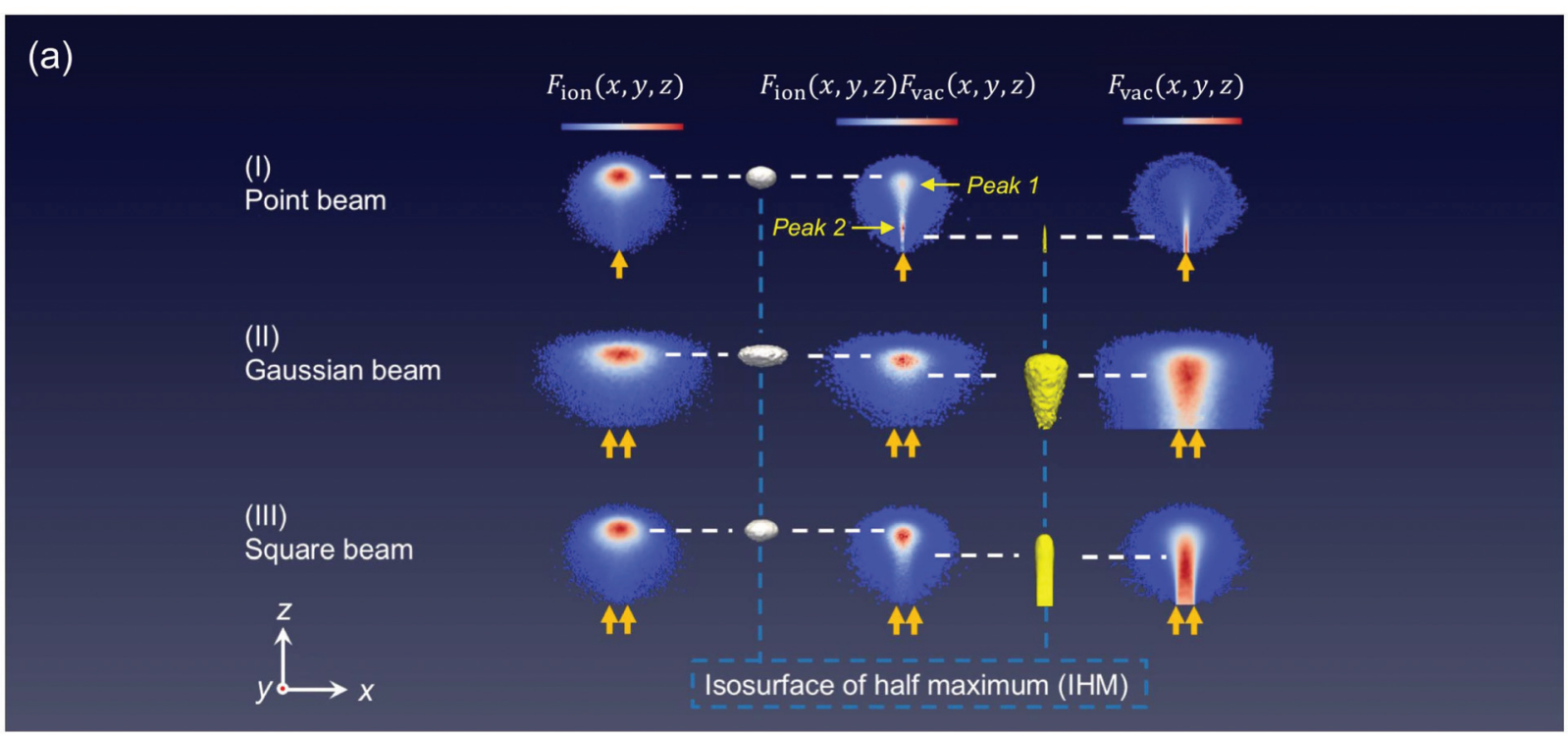

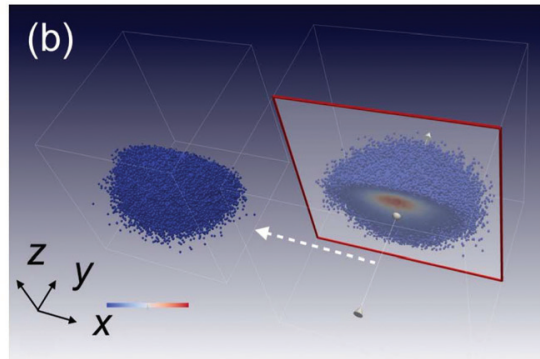

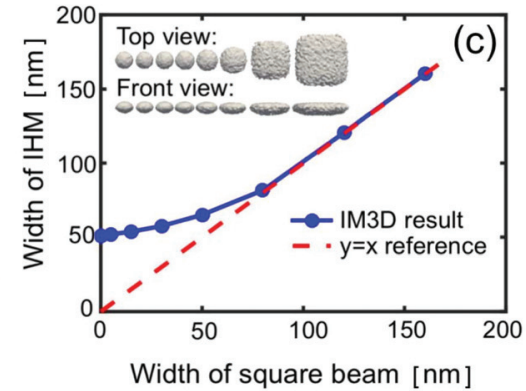

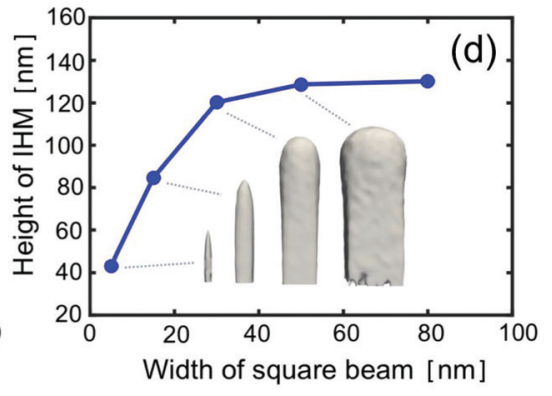

(f) (e)

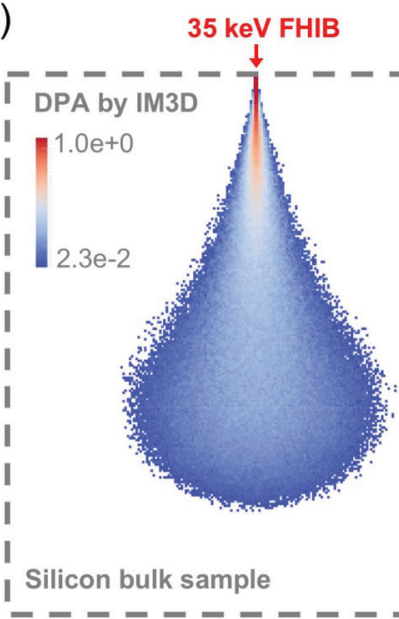

$35 \mathrm{keV}$ FHIB

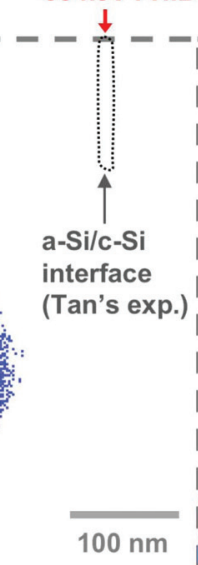

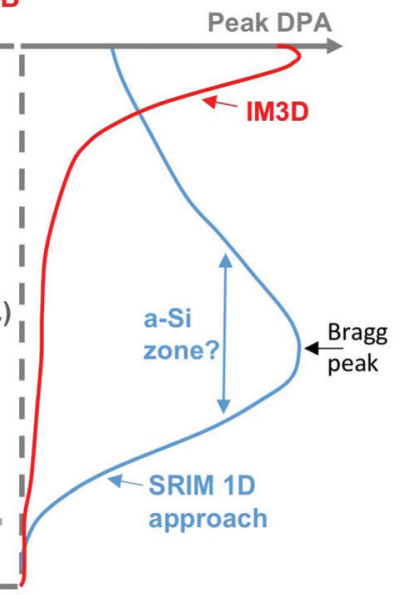

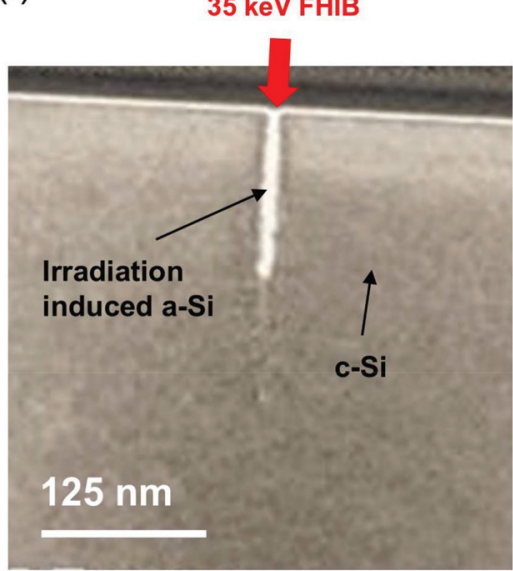

Fig. 6 (a) Sectional views of $F_{\text {ion, }} F_{\mathrm{vac}}(x, y, z)$ and $F_{\mathrm{ion}}(x, y, z) F_{\mathrm{vac}}(x, y, z)$ for a point beam, a Gaussian beam $(\sigma=30 \mathrm{~nm})$, and a square beam (30 nm width). The IHM of ${ }^{15} \mathrm{~N}^{+}$ions and vacancies are shown, which directly reflect the FWHM in 3D. The white dashed lines point out the peak position in $F_{\text {ion }}$ and $F_{\mathrm{vac}}$. (b) A cross section cut from (a), parallel to the $x-z$ plane. The ions are injected at the center of the bottom along the $z$-axis. (c) Evolution of the IHM of injected ions (from a circle to a square, via top view) as the square beam size grows, showing changes in the $x-y$ plane but not along the $z$-axis. Top and front views refer to the $-z$ and $+y$ viewing directions, respectively. (d) Evolution of the IHM of vacancies as the square beam size grows, showing changes along the $x, y$, and $z$ axes. Its height asymptotically reaches a saturation value, which is close to the height of IHM of injected ions. (e) A highly radiation damaged zone at the entrance (as shown by the 2D distribution and the red solid line), predicted by IM3D simulation of a $35 \mathrm{keV}$ nano-beam of $\mathrm{He}^{+}$ions into the silicon bulk sample, matches very well with the irradiation induced amorphous regions enclosed by the black dotted line (Tan et al.'s experiment ${ }^{47}$ ). On the other hand, the DPA calculated by the SRIM 1D approach (blue solid line) predicts a highly-damaged region much deeper in the sample. (f) TEM bright-field image ${ }^{47}$ of silicon after a line-exposure of $35 \mathrm{keV}^{\mathrm{He}} \mathrm{e}^{+}$irradiation with a dose of $2.6 \times 10^{4}$ ions per $\mathrm{nm}$ by Tan et al. Reprinted with permission from ref. 47. Copyright AIP Publishing LLC. 
1 quite close to the peak of the injected dopants. Similar to the incorrect prediction of the $z_{\max }$ of DV (eqn (4) and (7) give totally different results, as shown in Fig. 5), our 3D simulation reveals two $3 \mathrm{D}$ peaks in $F_{\text {ion }} F_{\text {vac }}$, while the $1 \mathrm{D}$ simulation predicts only one peak at a very different position. The 'needle' shape of $\operatorname{IHM}\left(F_{\text {vac }}\right)$ in contrast to the spherical shape of $\operatorname{IHM}\left(F_{\text {ion }}\right)$ explains why the relative error of the 1D approach for $F_{\mathrm{vac}}$ is several orders of magnitude smaller than that of $F_{\text {ion }}$ (see Fig. 2b and 3d).

For a Gaussian beam with $\sigma=30 \mathrm{~nm}, F_{\text {ion }}$ and $F_{\text {vac }}$ take on nut-shapes. $\operatorname{IHM}\left(F_{\text {ion }}\right)$ shares the same height (along the $z$-axis) as that of the point source case, while its lateral width is higher. On the other hand, $\operatorname{IHM}\left(F_{\text {vac }}\right)$ has a 'drumstick' shape, and overlaps with $\operatorname{IHM}\left(F_{\text {ion }}\right)$. It leads to a single peak of $F_{\text {ion }} F_{\text {vac }}$, proportional to the ${ }^{15} \mathrm{~N}-\mathrm{V}$ recombination rate. The 'drumstick' shape, which marks the lateral expansion of the FWHM as ions penetrate deeper, is caused by a higher ion density at the entrance when approaching the beam center for a Gaussian distribution. Thus, as the high-density ions move farther, they will scatter laterally and join the low-density ions originating from the ring of the Gaussian beam at the entrance. As such, the drumstick shape actually contains the beam profile information, which can be attributed to a convolution effect. Since simulations of each ion are independent (in other words, incoherent) in a Monte Carlo simulation, the distribution is a linear superposition. Thus, we can define the defect distribution $F_{\text {point }}(x, y, z)$ by a point beam ion source to be the point spread function (PSF). ${ }^{48}$ Then $F(x, y, z)$ by a monoenergetic beam with arbitrary ion density distribution $S(x, y)$ at the entrance is given by the convolution of $F_{\text {point }}(x, y, z)$ and $S(x, y)$ :

$$
F(x, y, z)=F_{\text {point }}(x, y, z)^{*} S(x, y)
$$

where * denotes mathematical convolution, and $S(x, y)$ in this case is a $2 \mathrm{D}$ Gaussian function with $\left(x_{0}, y_{0}\right)=(0,0)$ and $\sigma=$ $30 \mathrm{~nm}$. As the point beam grows into a larger Gaussian beam, we see a transition of $\operatorname{IHM}\left(F_{\text {vac }}\right)$ from a 'needle' to a 'drumstick' shape.

A square, uniformly distributed beam with width $2 \sigma$ :

$$
S(x, y)=S_{0}=\text { constant }, \quad|x|,|y| \leq \sigma
$$

approximates some real situations of the ion irradiation experimenth with masks. Fig. 6c and d show the evolution of the IHM as $\sigma$ increases. Looking from the front view $(+y$ direction), the thickness and height of $\operatorname{IHM}\left(F_{\text {ion }}\right)$ remain stable, while from the top view ( $-z$ direction), its lateral shape (in the $x-y$ plane) evolves from a round shape into a square, and its size increases. The curve in Fig. 6c shows that its lateral width starts at $50 \mathrm{~nm}$ for a point beam, and slowly increases in speed until it catches up to the expansion of $\sigma$. Note that even when $\sigma$ is infinitely $\operatorname{small}, \operatorname{IHM}\left(F_{\text {ion }}\right)$ is still $50 \mathrm{~nm}$ wide due to blurring via scattering (i.e., point spreading). Here we denote this characteristic IHM width as $W_{\mathrm{IHM}}$ (apparently $<L_{\mathrm{S}}$ ). When $\sigma$ is above $80 \mathrm{~nm}$, the IHM of ions becomes very close to a square plate, and its size is almost the same as $\sigma$. This demonstrates a strong nano-size effect, at a characteristic scale around $50 \mathrm{~nm}$. It is also interesting to find that the IHM of vacancies looks like an obelisk, which grows taller as $\sigma$ increases. The top of ebelisk will slowly approach $\operatorname{IHM}\left(F_{\text {ion }}\right)$, until it finally touches it at $\sigma=128 \mathrm{~nm}$. The top of $\operatorname{IHM}\left(F_{\text {vac }}\right)$ gets blunter as well. It is clearly shown in the simulations that the center line $(x=0, y=$ 0 ) approaches the $1 \mathrm{D}$ approach ahen $_{\mathbf{2}} \gg L_{\mathrm{S}}>W_{\mathrm{IHM}}$, since:

$$
\begin{gathered}
\lim _{\sigma \gg W_{\mathrm{IHM}}} F(x=0, y=0, z)=\lim _{\sigma \gg W_{\mathrm{IHM}}}\left(F_{\text {point }}{ }^{*} S\right)(x=0, y=0, z) \\
=\lim _{\sigma \gg W_{\mathrm{IHM}}} S_{0} \iint_{|u|,|v| \leq \sigma} F_{\text {point }}(0-u, 0-v, z) \mathrm{d} u \mathrm{~d} v \\
=S_{0} \iint_{-\infty}^{\infty} F_{\text {point }}(u, v, z) \mathrm{d} u \mathrm{~d} v=S_{0} f(z)
\end{gathered}
$$

Lastly, we point to a striking effect that for broad beams, the maximum radiation dose is found deep inside the material and away from the surface, so radiation damage effects like amorphization $^{49}$ and helium bubbles ${ }^{50}$ are seen to occur quite a distance away from the surface (the "Bragg peak" effect with broad beams). To demonstrate the nanobeam effect in contrast to a broad beam, we also performed a simulation similar to Tan et al.'s experiment: ${ }^{47}$ a $35 \mathrm{keV}$ focused helium ion beam (FHIB) with $\sigma$ around $1 \mathrm{~nm}$ entered a silicon single-crystal sample with the beam direction perpendicular to the surface. The simulation results of DPA distributions by IM3D and the 1D approach are compared in Fig. 6e. The peak DPA (plotted as red and blue curves in Fig. 6e) is defined as the maximum DPA for a fixed depth $z$. It is found that the peak damage zone is very close to the entrance surface with the nanobeam, instead of the deeper damage peak found using the 1D approach. Because high-dose irradiation leads to a crystal-silicon (c-Si) $\rightarrow$ amorphous silicon (a-Si) transition, IM3D and the 1D approach will predict the formation of the a-Si zone at very different depths in the case of the narrow nanobeam. Previous broad-beam ion implantation experiments ${ }^{49}$ have shown that the initiation of an a-Si zone occurs at a position deeper than the peak of the DPA curve as calculated by the 1D approach, while a large c-Si zone still exists at the surface with the following layer structure: surface $\rightarrow \mathrm{c}-\mathrm{Si} \rightarrow \mathrm{a}-\mathrm{Si} \rightarrow \mathrm{c}-\mathrm{Si}$. In contrast, post-irradiation transmission electron microscopy (TEM) characterization of the ${ }^{4} \mathrm{He}^{+}$nanobeam irradiated silicon ${ }^{47}$ (Fig. 6f) demonstrates the generation of an a-Si zone right at the surface $(z \rightarrow 0)$, matching the IM3D predictions. In other words, the most conspicuous radiation zone (resembled by the various IHMs) will entertain deformations and even topological transitions as one varies $\sigma$, even with just simple flat surfaces. More complex transitions may occur when the nano-target effect is added.

SRIM can also perform limited 3D simulations. It can deal with beam shapes (necessary for nano-beam simulations) by the convolution method but its accuracy is often insufficient because of low meshing resolution/view-field width in the Monte Carlo tally (more details are shown in ESI S3†). Because SRIM is close-sourced, improvement of this tally resolution is difficult. IM3D adopts the high quality database of stopping 
powers of SRIM; however, it already supports beam shapes and adaptive meshing directly, and its physics engine is opensource. Both SRIM and IM3D can alse handle multilayer structures, but only IM3D can consider arbitrary, laterally nonhomogeneous 3D geometries. IM3D is therefore better suited to treat more complex nano-target geometries, such as irradiating nano-mushroom pillars (experiment from ref. 43, IM3D simulation from ref. 20), ion irradiation of nanotubes ${ }^{29}$ (ions will leak from one boundary and re-enter at another boundary), and even complex rough surfaces. ${ }^{51}$

Although our work focuses on the effects of beam and target size on the point defect distribution by an ion beam, we want to point out that the target size ${ }^{38,52}$ surface roughness $\Lambda^{51}$ and point defect concentration along the ion incident direction $^{53}$ will also have impacts on sputtering. For example, sputtering can be enhanced for nanostructured materials. ${ }^{52}$ As sputtering will modify the shape/size of targets, a full-3D simulation model that can consider dynamic nanostructure evolution under ion radiation will be necessary to address the defect distributions more accurately.

\section{Conclusions}

A precise estimation of point defect distributions by nanoscale ion implantation is not only vital for bridging the gap between bulk and nanoscale experiments, but also for fabrieating reproducible nanoscale devices. For many nanoscience and nanotechnology applications of ion irradiation where features including the beam size and/or sample size are below one micron, full-3D simulations and 3D mathematical descriptions of the radiation damage and implanted ion fields become absolutely necessary. The practice of using a 1D approach for these problems is highly suspicious, often incurring errors anywhere from $10 \%$ to $1000 \%$.

\section{Conflicts of interest}

The authors declare no competing financial interest.

\section{Author contributions}

Y. Y. and J. L. conceived the project. Y. Y. performed the simulations. M. P. S. and Y. Y. conceived the relative error analysis method. All authors analyzed the data and contributed to the discussion. Y. Y., M. P. S., and J. L. wrote the manuscript.

\section{Data availability}

The data that support the findings of this study are available from the corresponding authors on request.

\section{Acknowledgements}

We acknowledge the support from the United States National Science Foundation Grant No. DMR-1120901. The computational time on the Extreme Science and Engineering Discovery Environment (XSEDE) under the grant number TG-DMR130038 is gratefully acknowledged. Y. G. Li acknowledges the support by the National Natural Science Foundation of China (no. 11475215 and 11775254) and the Youth Innovation Promotion Association of CAS (no. 2016386). The authors thank Prof. Daniel Kiener from Montanuniversität

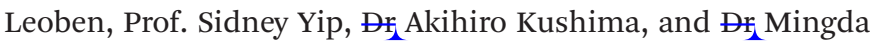
$\mathrm{Li}$, and $\mathrm{Mr}_{\mathrm{J}}$ Jiabao Zheng from MIT for helpful discussions.

\section{References}

1 I. Bayn, E. H. Chen, M. E. Trusheim, L. Li, T. Schröder, O. Gaathon, M. Lu, A. Stein, M. Liu, K. Kisslinger, H. Clevenson and D. Englund, Nano Lett., 2015, 15, 17511758.

2 T. Shinada, S. Okamoto, T. Kobayashi and I. Ohdomari, Nature, 2005, 437, 1128-1131.

3 P. Maletinsky, S. Hong, M. S. Grinolds, B. Hausmann, M. D. Lukin, R. L. Walsworth, M. Loncar and A. Yacoby, Nat. Nanotechnol., 2012, 7, 320-324.

4 S. Hoffmann, J. Bauer, C. Ronning, T. Stelzner, J. Michler, C. Ballif, V. Sivakov and S. H. Christiansen, Nano Lett., 2009, 9, 1341-1344.

5 J. F. Ziegler, M. D. Ziegler and J. P. Biersack, Nucl. Instrum. Methods Phys. Res., Sect. B, 2010, 268, 1818-1823.

6 G. S. Was, Z. Jiao, E. Getto, K. Sun, A. M. Monterrosa, S. A. Maloy, O. Anderoglu, B. H. Sencer and M. Hackett, Scr. Mater., 2014, 88, 33-36.

7 B. Grotz, M. V. Hauf, M. Dankerl, B. Naydenov, S. Pezzagna, J. Meijer, F. Jelezko, J. Wrachtrup, M. Stutzmann, F. Reinhard and J. A. Garrido, Nat. Commun., 2012, 3, 729.

8 F. Watt, M. B. H. Breese, A. A. Bettiol and J. A. Van Kan, Mater. Today, 2007, 10, 20-29.

9 T. C. Pekin, F. I. Allen and A. M. Minor, J. Microsc., 2016, 264, 59-63.

10 Y. Yao, M. W. van Mourik, P. Santhana Raman and J. A. van Kan, Nucl. Instrum. Methods Phys. Res., Sect. B, 2013, 306, 265-270.

11 N. P. Economou, J. A. Notte and W. B. Thompson, Scanning, 2012, 34, 83-89.

12 S. Tan, R. Livengood, P. Hack, R. Hallstein, D. Shima, J. Notte and S. McVey, J. Vac. Sci. Technol., B: Nanotechnol. Microelectron.: Mater., Process., Meas., Phenom., 2011, 29, $06 \mathrm{~F} 604$.

13 D. M. Toyli, C. D. Weis, G. D. Fuchs, T. Schenkel and D. D. Awschalom, Nano Lett., 2010, 10, 3168-3172.

14 S. Pezzagna, D. Rogalla, H. W. Becker, I. Jakobi, F. Dolde, B. Naydenov, J. Wrachtrup, F. Jelezko, C. Trautmann and J. Meijer, Phys. Status Solidi A, 2011, 208, 2017-2022. 
15 S. Pezzagna, D. Wildanger, P. Mazarov, A. D. Wieck, Y. Sarov, I. Rangelow, B. Naydenov, F. Jelezko, S. W. Hell and J. Meijer, Small, 2010, 6, 2117-2121.

16 D. Scarabelli, M. Trusheim, O. Gaathon, D. Englund and S. J. Wind, Nano Lett., 2016, 16, 4982-4990.

17 Proceedings of the 7th International Workshop: Microbeam Probes of Cellular Radiation Response, Radiation Research Society, 2006, vol. 166, pp. 652689.

18 C. Borschel and C. Ronning, Nucl. Instrum. Methods Phys. Res., Sect. B, 2011, 269, 2133-2138.

19 W. Möller, Nucl. Instrum. Methods Phys. Res., Sect. B, 2014, 322, 23-33.

20 Y. G. Li, Y. Yang, M. P. Short, Z. J. Ding, Z. Zeng and J. Li, Sci. Rep., 2015, 5, 18130.

21 F. Schiettekatte and M. Chicoine, Nucl. Instrum. Methods Phys. Res., Sect. B, 2016, 371, 106-110.

22 S. Furukawa, H. Matsumura and H. Ishiwara, Jpn. J. Appl. Phys., 1972, 11, 134.

23 H. Runge, Phys. Status Solidi, 1977, 39, 595-599.

24 S. A. Rishton and D. P. Kern, J. Vac. Sci. Technol., B: Microelectron. Nanometer Struct. - Process., Meas., Phenom., 1987, 5, 135.

25 K. Mitsuishi, M. Shimojo, M. Takeguchi, M. Tanaka and K. Furuya, Jpn. J. Appl. Phys., 2006, 45, 5517-5521.

26 T. H. P. Chang, J. Vac. Sci. Technol., 1975, 12, 1271-1275.

27 M. Parikh, J. Appl. Phys., 1979, 50, 4371-4377.

28 H. Eisenmann, J. Vac. Sci. Technol., B: Microelectron. Nanometer Struct. - Process., Meas., Phenom., 1993, 11, 2741.

29 A. Felten, X. Gillon, M. Gulas, J. Pireaux, X. Ke, G. Van Tendeloo, C. Bittencourt, E. Najafi and A. P. Hitchcock, ACS Nano, 2010, 4, 4431-4436.

30 A. V. Krasheninnikov and K. Nordlund, J. Appl. Phys., DOI: 10.1063/1.3318261.

31 J. Li, D. Stein, C. McMullan, D. Branton, M. J. Aziz and J. A. Golovchenko, Nature, 2001, 412, 166-169.

32 C. Dai and J. H. Cho, Nano Lett., 2016, 16, 3655-3660.

33 P. Nukala, C.-C. Lin, R. Composto and R. Agarwal, Nat. Commun., 2016, 7, 10482.

34 D. C. Dillen, K. Kim, E. Liu and E. Tutuc, Nat. Nanotechnol., 2014, 9, 116-120.

35 J. Cardellino, N. Scozzaro, M. Herman, A. J. Berger, C. Zhang, K. C. Fong, C. Jayaprakash, D. V. Pelekhov and P. C. Hammel, Nat. Nanotechnol., 2014, 9, 343-347.
36 J. T. Muhonen, J. P. Dehollain, A. Laucht, F. E. Hudson, R. Kalra, T. Sekiguchi, K. M. Itoh, D. N. Jamieson, J. C. McCallum, A. S. Dzurak and A. Morello, Nat. Nanotechnol., 2014, 9, 986-991.

37 C. Gómez-Navarro, P. J. de Pablo, J. Gómez-Herrero, B. Biel, F. J. Garcia-Vidal, A. Rubio and F. Flores, Nat. Mater., 2005, 4, 534-539.

38 A. Johannes, H. Holland-Moritz and C. Ronning, Semicond. Sci. Technol., 2015, 30, 33001.

39 C. Borschel, M. E. Messing, M. T. Borgström, W. Paschoal, J. Wallentin, S. Kumar, K. Mergenthaler, K. Deppert, C. M. Canali, H. Pettersson, L. Samuelson and C. Ronning, Nano Lett., 2011, 11, 3935-3940.

40 W. Möller, A. Johannes and C. Ronning, Nanotechnology, 2016, 27, 175301.

41 A. V. Krashepinnilkov and K. Nordlund, J. Appl. Phys., 2010, 107, 71301.

42 L. Pelaz, L. A. Marqués, M. Aboy, P. López and I. Santos, Mater. Sci. Semicond. Process., 2017, 62, 62-79.

43 R. Liontas, X. W. Gu, E. Fu, Y. Wang, N. Li, N. Mara and J. R. Greer, Nano Lett., 2014, 14, 5176-5183.

44 D. Kiener, P. Hosemann, S. A. Maloy and A. M. Minor, Nat. Mater., 2011, 10, 608-613.

45 M. P. Short, D. R. Gaston, M. Jin, L. Shao and F. A. Garner, J. Nucl. Mater., 2015, 1-8.

46 T. Faney and B. D. Wirth, Model. Simul. Mater. Sci. Eng., 2014, 22, 65010.

47 S. Tan, K. Klein, D. Shima, R. Livengood, E. Mutunga and A. Vladár, J. Vac. Sci. Technol., B: Nanotechnol. Microelectron.: Mater., Process., Meas., Phenom., 2014, 32, 06FA01.

48 D. Winston, J. Ferrera, L. Battistella, A. E. Vladár and K. K. Berggren, Scanning, 2012, 34, 121-128.

49 M. Ishimaru, S. Harada and T. Motooka, J. Appl. Phys., 1997, 81, 1126-1130.

50 M. S. Ding, J. P. Du, L. Wan, S. Ogata, L. Tian, E. Ma, W. Z. Han, J. Li and Z. W. Shan, Nano Lett., 2016, 16, 41184124.

51 Y. Li, Y. Yang, M. P. Short, Z. Ding, Z. Zeng and J. Li, Nucl. Fusion, 2017, 57, 16038.

52 A. Johannes, S. Noack, W. Paschoal, S. Kumar, D. Jacobsson, H. Pettersson, L. Samuelson, K. a. Dick, G. Martinez-Criado, M. Burghammer and C. Ronning, J. Phys. D: Appl. Phys., 2015, 48, 79501.

53 G. Hobler, R. M. Bradley and H. M. Urbassek, Phys. Rev. B, 2016, 93, 205443. 